\title{
CCN Forwarding Engine Based on Bloom Filters
}

\author{
M. Tortelli, L. Alfredo Grieco and G. Boggia \\ DEE - Politecnico di Bari Italy \\ \{m.tortelli,a.grieco,g.boggia\}@poliba.it
}

\begin{abstract}
The lack of scalable routing algorithms is one of the main obstacles that slow down a large deployment of Content Centric Networking on an Internet-scale. From one side, content based networking promises to solve the current problems of the Internet. On the other hand, instead, it requires routers to account for a very huge amount of content names. Bloom Filters are widely recognized as a possible solution to this limitation. At the same time, their adoption requires careful tuning rules and novel design methodologies. In this perspective, the present contribution proposes a Bloom Filter-based routing scheme for Content Centric Networking (CCN) and shows several preliminary observations about Bloom Filters size and signaling overhead.
\end{abstract}

\section{Categories and Subject Descriptors}

C.2.1 [Computer-Communication Networks]: Network Architecture and Design; C.2.2 [Computer-Communication Networks]: Network Protocols.

\section{Keywords}

CCN, Forwarding, Bloom Filter.

\section{INTRODUCTION}

$\mathrm{CCN}$ is a promising architecture for the Future Internet [2], devised to face the upcoming "Zettabyte Era" [1]. Based on only two types of packets, i.e., Interest and Data, it advocates three pivotal points: every node is content-aware; routing and forwarding strategies are based on hierarchical content names, and are applied only to Interests; contents can be retrieved by every node, thanks to distributed caching operations. However, renouncing IP addresses will force every node to keep track, in its Forwarding Information Base (FIB), of a huge amount of contents available on the web (e.g., Google reports a lower bound of $10^{12}$ unique URLs [4]). In this eventuality, lookup times will degrade forwarding performances.

Permission to make digital or hard copies of all or part of this work for personal or classroom use is granted without fee provided that copies are not made or distributed for profit or commercial advantage and that copies bear this notice and the full citation on the first page. To copy otherwise, to republish, to post on servers or to redistribute to lists, requires prior specific permission and/or a fee.

CFI'12, September 11-12, 2012, Seoul, Korea

Copyright 2012 ACM 978-1-4503-1690-3/12/09 ...\$15.00.
In this work, we present our vision about the use of Bloom Filters (BFs) in a CCN forwarding engine. With respect to proposals in [3] and [7], we seek for an optimized use of BFs for Internet routing, minimizing signaling overhead and flooded Interests. In particular, exploiting the hierarchical structure of content names and the content awareness of every node, we propose the use of two types of BFs for each node interface: a large one (not exchangeable between nodes) and a small one. We, also, provide some preliminary observations about the exchange times of update messages between nodes to keep the BFs consistent.

\section{EVALUATION OF UPDATE TIMES}

A Bloom Filter [6] is a data structure conceived to efficiently perform membership queries on a large data set. In particular, using an array of $m$ bits, all initially set to 0 , we can map each item to the corresponding "footprint", i.e., every ID is hashed using $k$ different hash functions to obtain the positions of the $k$ bits that will be set to 1 . But, collisions in the mapping phase can lead to false positives.

In a CCN scenario, nodes can exchange BFs representing contents in their cache, thus every one is aware of contents in the neighborhood. The collected information can be used to limit flooded Interests, selecting the interface whose BF provides a match. The problem is that these BFs need to be updated in order to limit the false positives caused by stale informations (neighbors may have replaced the contents previously advertised). Hence, in a real scenario, every node must periodically send its updated BF, potentially leading to an uncontrolled occupation of the links bandwidth.

To estimate these update times, we run simulations based on the model presented in [5] for a general network of caches with a Least Recently Used (LRU) replacement policy. We used: a random graph with 50 nodes, 150 links and an average path length of 3.22 hops; a content catalog of about $N=10^{4}$ contents with Zipf-like probability (Zipf parameter, $\alpha=0.65)$, and a cache size of $B=500$ contents for every node $(B / N=0.05)$. Thanks to the aforementioned model, we extracted the overall incoming requests rates (made up of exogenous requests and misses from the neighbors) and the interarrival times (supposed exponentially distributed) for each content at every node. Then, starting from a randomly initialized full cache (simulating a steady state regime), we imposed that an update message would have been sent upon the change of a predefined number of contents in the cache (we used three different thresholds, 10\%, 20\% and 30\% of the cache size), thus obtaining the rates reported in Tab. 1.

The short times reported in the first column, obtained 
Table 1: Update Times

\begin{tabular}{|c|c|c|c|}
\hline Threshold & Min [s] & Avg [s] & Max [s] \\
\hline $10 \%$ & 496 & 1004 & 1152 \\
\hline $20 \%$ & 630 & 1404 & 1512 \\
\hline $30 \%$ & 745 & 1692 & 1800 \\
\hline
\end{tabular}

from a central node with twelve interfaces, indicate that a size reduction of the BFs exchanged between nodes, would be of great help in limiting the overhead caused by signaling traffic. Indeed, this is a key feature of our CCN forwarding scheme, which is described in the following.

\section{BF-BASED FORWARDING ENGINE}

We propose the adoption of two types of BFs for each interface: a large one and a small one. The former is a Stable Bloom Filter (SBF) [6], able to randomly delete stale information; it is not exchanged between nodes and it is updated according to the entire name of contents retrieved from the corresponding interface. Furthermore, it is dimensioned considering $N=10^{6}$ contents (a reasonable number in an ISP-scale network [4]), and a probability of false positive, $P_{f p}$, equal to $0.1 \%$. Considering that every cell of the filter is a $d=3$ bit counter, the size of the filter will be $M \approx 5.4$ MByte (according to the formula in [6]).

The only way to reduce the size of a $\mathrm{BF}$, with a fixed $P_{f p}$, is to account for a smaller number of contents. In a CCN scenario, this means a hierarchical name aggregation. As observed in [4], a regulation on the content name length and on their hierarchical structure, would be very beneficial. Following this road, we propose every node mapping the contents in their cache on separated BFs for each name field (see Fig. 1) until a tunable name depth, $L$.

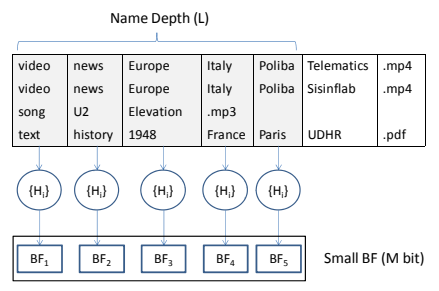

Figure 1: Mapping Procedure.

Making this, the population to be considered for each field becomes considerably smaller. For example, accounting for $n=2500$ possible unique names for each field and imposing $P_{f p}=0.05 \%$, we obtain a BF of size $m \approx 5 \mathrm{kByte}$. Hence, the size of our small BF, made up of $L$ independent small BFs, will be, for example, equal to $M=50 \mathrm{kByte}$ (if we consider a name depth of $L=10)$. This is smaller than the BF presented above (i.e., $M=5.4$ MByte), so that it could be efficiently excahanged between nodes, without overloading the network links with updates.

A certain level of inaccuracy is the price to pay with name aggregation; nevertheless, the partial matching of content names enables nodes to implement a sort of greedy routing policy aimed at limiting flooded Interests. In addition, we are able to compensate for this inaccuracy due to the complete information hold in the larger BFs.

Indeed, in our forwarding strategy (see Fig. 2), the SBFs will be the first to be queried upon the reception of an In- terest (provided that neither the requested content is in the cache nor an identical Interest has been previously sent). Then, if this query fails or if the Interest is not satisfied

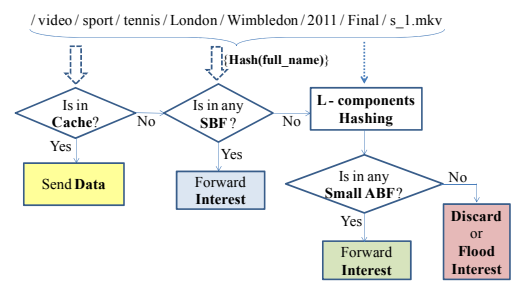

Figure 2: Forwarding Scheme.

after a timeout, the node proceeds with the hashing of the $L$ fields (separately) of the content name. At this point we can implement either a keyword-based membership query, thus forwarding the Interest towards the interface that provides the maximum number of matches on $L$ (independently of their order in the entire name), or perform the membership query in sequence, starting from the the first field $(l=1)$ and continuing, in case of positive matches, until the $L$-th field. The Interest will be forwarded toward the interface that provides the match with the highest value of $l$.

\section{FUTURE WORK}

As future work, we plan to develop a customizable CCN simulator to finely characterize the behavior of the two-level forwarding strategy we propose. Particular attention will be devoted to the analysis of scalability issues, which could arise in the presence of very large content catalogs (with cardinality much greater then $10^{6}$ contents), as well as to the effects of aggregation and propagation of BF updates.

\section{ACKNOWLEDGMENTS}

This work was supported by the project ERMES PON01 $03113 / 3$, founded by the Italian MIUR.

\section{REFERENCES}

[1] Cisco visual networking index: Forecast and methodology, 2010-2015. White Paper, Jun. 2011.

[2] V. Jacobson, D. K. Smetters, J. D. Thornton, M. F. Plass, N. H. Briggs, and R. L. Braynard. Networking named content. In Proc. of CONEXT, Dec. 2009.

[3] M. Lee, K. Cho, K. Park, T. Kwon, and Y. Choi. SCAN: Scalable content routing for content-aware networking. In Proc. of IEEE ICC, Jun. 2011.

[4] D. Perino and M. Varvello. A reality check for content centric networking. In Proc. of ACM SIGCOMM ICN Workshop, Aug. 2011.

[5] E. J. Rosensweig, J. Kurose, and D. Towsley. Approximate models for general cache networks. In Proc. of IEEE INFOCOM, Mar. 2010.

[6] S. Tarkoma, C. E. Rothenberg, and E. Lagerspetz. Theory and practice of bloom filters for distributed systems. IEEE Communication Surveys Tutorials, 14(1), quarter 2012.

[7] Y. Wang, K. Lee, B. Venkataraman, R. L. Shamanna, I. Rhee, and S. Yang. Advertising cached contents in the control plane: Necessity and feasibility. In Proc. of IEEE INFOCOM NOMEN, Mar. 2012. 